Recebido em 11/2019. Aceito para publicação em 12/2019.

\title{
COMPÓSITOS DE MADEIRA PLÁSTICA/CARGA INORGÂNICA: INFLUÊNCIA DO TIPO DE CARGA
}

\section{PLASTIC WOOD/INORGANIC FILLER COMPOSITES: INFLUENCE OF FILLER TYPE}

\author{
Alice Souza Gonçalves ${ }^{1}$ \\ Camila Ferreira Gerardo ${ }^{2}$ \\ Silvia Cristina Alves França ${ }^{3}$ \\ Daniele Cruz Bastos ${ }^{4}$ \\ Shirleny Fontes Santos 5
}

\begin{abstract}
Resumo: O presente trabalho teve como objetivo o estudo da influência da adição de dois tipos de cargas inorgânicas, a sílica $\left(\mathrm{SiO}_{2}\right)$ e a mica muscovita, na obtenção de compósitos com resíduos de madeira plástica pós-industrial. Sendo a carga de mica, um produto natural, oriundo de mineração e a sílica, obtida por uma rota de síntese química (Método Pechini). Os materiais compósitos foram obtidos em extrusora dupla-rosca, prensados, para obtenção de filmes planos e posteriormente caracterizados por análises de densidade, dureza Shore $D$, índice de fluidez (MFI) e microscopia óptica. Os resultados indicam que a adição de até $10 \%$ de muscovita não modificou as propriedades da matriz polimérica, entretanto, a adição de $10 \%$ de sílica provocou uma redução nos valores de densidade, dureza e MFI, o que pode estar relacionado a uma fraca interação polímero-carga. Pelos espectros de FTIR pode-se inferir que não há interação química entre a matriz e as cargas utilizadas.
\end{abstract}

Palavras-chave: Método Pechini; compósito polimérico; carga inorgânica.

Abstract: The aim of the present work was to study the influence of the addition of two types of inorganic fillers, silica $\left(\mathrm{SiO}_{2}\right)$ and muscovite mica, to obtain composites with post-industrial plastic wood residues. Mica is a natural product, derived from mining, and silica is obtained by a chemical synthesis route (Pechini Method). The composite materials were obtained in double screw extruder, pressed to obtain films and later characterized by density analysis, Shore D hardness, melt flow index (MFI) and optical microscopy. The results indicate that the addition of up to $10 \%$ of muscovite did not modify the properties of the polymeric matrix; however, the addition of $10 \%$ silica caused a reduction in density, hardness and MFI values, which may be related to poor polymer-filler interaction. From the FTIR spectra, it can be inferred that there is no chemical interaction between the matrix and the fillers used.

Keywords: Pechini Method; polymeric composite; inorganic load.

\section{INTRODUÇÂO}

Desde seu advento, os polímeros vêm promovendo impactantes alterações nas sociedades em seus amplos setores, graças à diversidade de produtos, com designers inovadores e de fácil obtenção, entre outras vantagens. Entretanto, o crescente consumo destes materiais tem levado à produção de uma quantidade enorme de

\footnotetext{
${ }^{1}$ Centro Universitário Estadual da Zona Oeste (UEZO), Brasil. E-mail: alice.souzag@outlook.com.

${ }^{2}$ Centro Universitário Estadual da Zona Oeste (UEZO), Brasil. E-mail: camilafgerardo@gmail.com.

${ }^{3}$ Centro Universitário Estadual da Zona Oeste (UEZO), Brasil. E-mail: sfranca@cetem.gov.br.

${ }^{4}$ Centro Universitário Estadual da Zona Oeste (UEZO), Brasil. E-mail: daniele_cruz@yahoo.com.br.

${ }^{5}$ Centro Universitário Estadual da Zona Oeste (UEZO), Brasil. E-mail: lenyfontes@yahoo.com.br.
} 
resíduos pós-consumo que causam grande impacto ambiental (CRUZ et al, 2011).

Uma das medidas mais utilizadas para amenizar esse impacto é a reciclagem, em que os polímeros pós-consumo voltam à linha de processamento para obtenção de um novo produto (CRUZ et al, 2011; ROMÂO; FRANCO; BUENO, 2010).

A reciclagem ainda enfrenta entraves, como a falta de apoio às empresas recicladoras e as dificuldades quanto ao correto descarte e recolhimento do "lixo". Contudo, estudos científicos mostram que esta é uma alternativa viável, principalmente se forem encontradas soluções que promovam a melhoria das propriedades mecânicas e térmicas do reciclado (CRUZ et al, 2011; ROMÃO; FRANCO; BUENO, 2010; MATOS, 2015).

A Madeira Plástica é um material que se enquadra neste contexto, pois é, usualmente, fabricada a partir da reciclagem de vários tipos de plásticos, que são reprocessados e pigmentados para gerar um novo produto. Entre os polímeros utilizados o PEAD (polietileno de alta densidade) é encontrado em maior quantidade (MARTINS, 2017; REZENDE, 2015).

A Madeira Plástica encontra aplicações semelhantes às da madeira comum, podendo ser pregada, parafusada, rebitada ou colada. É um material considerado ecológico, já que além de ajudar no reaproveitamento do lixo plástico contribui também para diminuir o desmatamento indevido de nossas florestas. Também se destaca por absorver pouca umidade, apresentar boa durabilidade e ser imune a pragas, mofos e fungos (OLIVEIRA; COSTA, 2013).

Apesar da série de vantagens apresentada pela madeira plástica, pelo fato dela ser fabricada primordialmente por poliolefinas, que são materiais intrinsecamente mais facilmente combustíveis, há preocupação com a resistência térmica desses materiais, tendo sido encontrados registros da ocorrência de empenamento destes materiais durante o uso, devido, provavelmente, a sua exposição ao calor do sol e outras intempéries (MARTINS, 2017; REZENDE, 2015; DIAS; ALVAREZ, 2017).

Uma alternativa para melhoria da resistência térmica e mecânica da madeira plástica é o estudo da incorporação de cargas de reforço, ou seja, na forma de compósito. Há vários tipos de materiais que vêm sendo utilizados como cargas de reforço em polímeros, dentre os quais se destacam as fibras de vidro, carbonato de cálcio, as fibras vegetais (sisal, coco, juta, etc) e as micas e argilas (MARINELLI; MONTEIRO; AMBROSIO, 2008). As características finais dos compósitos dependem das propriedades das cargas (granulometria, geometria, etc) e das resinas utilizadas como matriz, bem como da proporção entre os materiais e capacidade de adesão (FELIX, 2002).

Focando na possibilidade de reforço com mica, é conhecida sua ação reforçadora em diversos sistemas poliméricos, graças principalmente as suas propriedades de isolamento térmico, reforço mecânico e ao fato de ser abundantemente encontrada no 
Brasil (MONSORES et al., 2017; SANTOS; ANDRADE; SAMPAIO, 2011; OMAR; AKIL; AHMAD, 2011). Por outro lado, muitos autores defendem o uso das cargas sintéticas, como a sílica, já que a síntese possibilita um maior controle das características finais do material, como microestrutura, tamanho de partícula e pureza, entre outras (FONSECA, 2015; CHENG et al, 2007).

\section{$1.1 \quad$ Objetivo}

O presente trabalho tem como objetivo estudar a influência da adição de dois tipos de cargas $\left(\mathrm{SiO}_{2}\right.$ e mica) na obtenção de compósitos com madeira plástica. Sendo a carga de mica, um produto natural, oriundo de mineração e a sílica obtida por uma rota de síntese química (Método Pechini).

\section{REVISÃO DA LITERATURA}

A busca bibliográfica sobre o tema polímero/mica exibe uma grande variante deste sistema, em que pode ser encontrado o uso das micas do tipo muscovita, sericita e flogopita, dentre outras, e argilas tais como bentonita, montmorilonita e atalpugita além de diversos tipos de matrizes poliméricas (UNO et al., 2009; RAMOS FILHO et al., 2005, RAMOS et al, 1993), porém os estudos com o sistema específico madeira plástica/muscovita é ainda incipiente (ALMEIDA, 2018).

\subsection{Compósitos com mica muscovita}

No trabalho de Escócio et al. (2011), a mica foi incorporada ao elastômero termoplástico de poliuretano e processada em misturador interno acoplado a reômetro de torque. A análise das propriedades mecânicas e térmicas dos compósitos obtidos mostraram ocorrência de boa interação entre a matriz polimérica e a mica além de aumento de estabilidade térmica em alguns dos estágios de degradação. A análise dinâmico-mecânica revelou também aumento da rigidez do compósito na região borrachosa do elastômero.

Entretanto, a obtenção de um sistema compósito não é simples, entre outros fatores é necessário que haja adesão entre a matriz e o material incorporado. O estudo de Nicolau (2017) abordou esta problemática com uso do compatibilizante PE-g-MAH (Polietileno graftizado com Anidrido Maleico) no desenvolvimento de um compósito de Polipropileno (PP) e Muscovita. A mica foi introduzida na proporção de 10\%, 15\% e 20\% e o compatibilizante $1 \%, 3 \%$ e $5 \%$. A análise de dureza Shore $D$ revelou uma tendência a diminuição com aumento do teor de PE-g-MAH sugerindo aumento da plasticidade do material.

Outra alternativa promissora para melhora da adesão entre a mica e um polímero é a realização de tratamentos químicos. No trabalho de Rashid (2011), mica do tipo muscovita foi submetida a um tratamento de troca iônica. Uma molécula de natureza orgânica (brometo de octadeciltrimetilamonio) foi trocada pelo íon potássio, com isso evidenciou-se grande melhora na adesão entre os compósitos de mica (10-40\%) e epóxi além de aumento na resistência a flexão e tenacidade à fratura. 
Verbeek (2002), estudou a mica do tipo flogopita para obtenção de compósito com polietileno de baixa densidade para aplicação como material substituinte da madeira. 0 autor verificou os melhores resultados em relação a melhoria de propriedades mecânicas com misturas compondo $20 \%$ de polietileno e $10 \%$ de ácido maleico anidro como agente promovedor de adesão. O módulo de elasticidade alcançou $895 \mathrm{MPa}$ e aumento da resistência ao impacto foi observado com aumento da concentração de polímero e do agente de adesão.

O sistema madeira plástica/muscovita foi estudado por Almeida (2018). A carga mineral utilizava um tamanho de partícula da ordem de $100 \mu \mathrm{m}$ e foram estudadas as proporções de 10, 15 e 20\%. Os compósitos foram obtidos por processamento em misturador interno. Verificou-se que com aumento da quantidade de muscovita a densidade e a dureza aumentaram e o índice de fluidez diminuiu, sugerindo ação reforçadora da mica na matriz polimérica. Foi possível observar também uma pequena melhora na resistência térmica dos compósitos.

\subsection{Compósitos com sílica}

Dentre as cargas sintéticas, obtidas por rotas químicas, o uso da sílica $\left(\mathrm{SiO}_{2}\right)$ e da alumina $\left(\mathrm{Al}_{2} \mathrm{O}_{3}\right)$ é de particular interesse, pois elas proporcionam em muitos casos redução dos custos e do peso dos compósitos obtidos, que são requisitos de fundamental importância nas aplicações de engenharia, além das melhorias nas propriedades mecânicas e térmicas (OMAR; AKIL; AHMAD, 2011; FONSECA, 2015).

Sabe-se que a granulometria, por exemplo, pode aumentar a interação entre os componentes se houver uma boa dispersão e isso pode ser obtido quando o incorporante apresenta baixo tamanho de partícula. Neste sentido, a síntese da fase incorporante por rotas químicas, como o método Sol-Gel, o método Pechini, entre outros, surge como uma alternativa para alcançar a granulometria adequada, entre outras características (LI-YU; DAE-EUN, 2011; FONSECA, 2015).

\subsection{O Método Pechini}

O Método Pechini (ou técnica do complexo polimérico) envolve a propriedade de certos ácidos carboxílicos de formar quelatos com vários cátions. Estes quelatos são poliesterificados quando aquecidos em álcool polihidroxi com a formação de uma resina de poliéster onde os íons são imobilizados e tratados termicamente para obtenção dos óxidos desejados. O processo promove a formação de pós com boa homogeneidade, baixo tamanho de partícula, alta pureza, baixo custo e em relativamente baixa temperatura de processamento (SANTOS; FRANÇA; OGASAWARA, 2007; FONSECA, 2015).

A pesquisa bibliográfica, especificamente, sobre o sistema $\mathrm{PEAD} / \mathrm{SiO}_{2}$ em que a sílica tenha sido obtida pelo Método Pechini, revelou-se escassa (FONSECA, 2015). Entretanto, a investigação desses sistemas e de outros similares contribui para o entendimento de características do sistema de interesse (PASSOS et al, 2011; PALZA; VERGARA; ZAPATA, 2011). 
Por exemplo, Passos et al. (2011) reportou em seu trabalho o preparo de nanocompósitos a base de poli(etileno-co-acetato de vinila) (EVA) e óxido de sílica $\left(\mathrm{SiO}_{2}\right)$ síntetico, com tamanho de partícula da ordem de $40 \mathrm{~nm}$. Os nanocompósitos foram caracterizados principalmente pela espectroscopia de ressonância magnética nuclear (RMN). Pelos dados de RMN foi observado que até cerca de $5 \%$ de sílica em massa obteve-se um nanocompósito polimérico com boa dispersão da sílica devido a uma forte interação entre a sílica e matriz de EVA.

Palza, Vergara e Zapata (2011) sintetizou nanopartículas de sílica esféricas pelo método sol-gel fundidas com uma matriz de polipropileno, a fim de quantificar o seu efeito sobre os comportamentos térmicos e mecânicos dos compósitos de polímero resultantes. Na microscopia de transmissão eletrônica, verificou-se que as nanopartículas esféricas ficaram dispersas na matriz de polímero. Na termogravimetria, as nanopartículas aumentaram a estabilidade à degradação térmica da matriz polimérica sob condições oxidativas. Ensaios de tração mostraram que os compósitos com nanopartículas esféricas podem exibir aumento do módulo elástico.

\section{MATERIAIS E MÉTODOS}

\subsection{Síntese da sílica $\left(\mathrm{SiO}_{2}\right)$ pelo Método Pechini}

Para a síntese de $\mathrm{SiO}_{2}$ pelo método Pechini, inicialmente, foi preparada solução alcoólica de ácido cítrico $1 \mathrm{~mol} / \mathrm{L}$ e aquecida a $70^{\circ} \mathrm{C}$ sob agitação. Quando essa temperatura foi atingida, adicionou-se ao meio reacional etilenoglicol e o TEOS (tetraetilortosilicato), que é o precursor de silício. A relação estequiométrica entre ácido cítrico e etilenoglicol foi de 2:1, em mol, e de ácido cítrico e o precursor de Si de 1:1/3, em mol. O sistema foi mantido em aquecimento por cerca de 6 horas até que se obtivesse um gel. Este gel foi inicialmente queimado a $500^{\circ} \mathrm{C}$ e em seguida calcinado a $1000^{\circ} \mathrm{C}$.

\subsection{Obtenção dos compósitos Madeira plástica/Carga inorgânica}

Os compósitos foram obtidos pela mistura de madeira plástica e SiO2 e madeira plástica e mica, conforme fotos apresentadas na Figura 1 (a, b, c).

Figura 1 - Fotos dos filmes de compósitos obtidos: (a) Madeira plástica pura (b) $\mathrm{MP} / \mathrm{SiO}_{2} 10 \%$ e (c) MP/Mica $10 \%$.
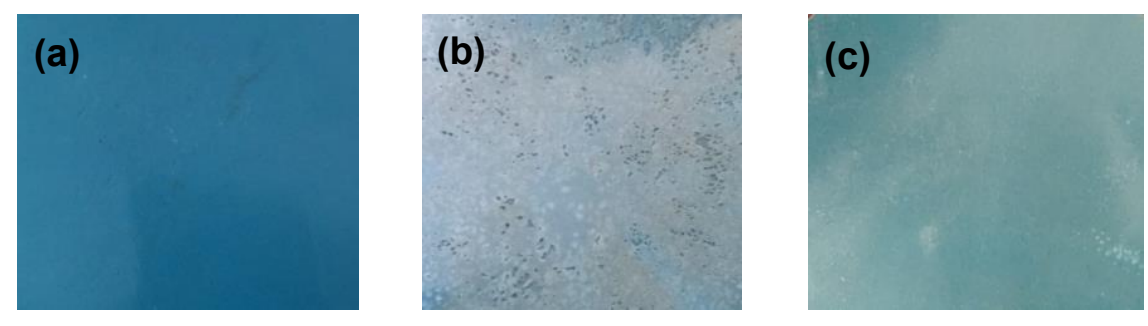

Fonte: As autoras.

A Madeira Plástica utilizada no trabalho é um resíduo do processo de confecção de móveis de praça, gentilmente cedido pela COMLURB (Companhia Municipal de 
Limpeza Urbana do Rio de Janeiro) e tem em sua composição $90 \%$ de polietileno de alta densidade, de acordo com Martins et al. (2017).

A mica do tipo muscovita, tamanho de partícula inferior a $100 \mu \mathrm{m}$, utilizada no trabalho, foi fornecida pelo CETEM (Centro de Tecnologia Mineral) e beneficiada conforme método descrito no trabalho de Monsores et al. (2017).

Foram preparadas misturas entre $\mathrm{MP} / \mathrm{SiO}_{2}$ e $\mathrm{MP} /$ Mica com diferentes percentuais da carga ( $3 \%$ e $10 \%$ em massa), de acordo com a Tabela 1. Os compósitos foram obtidos em extrusora dupla-rosca, co-rotatória com perfil de temperatura variando de 170 a $200^{\circ} \mathrm{C}$ e $90 \mathrm{rpm}$. As amostras foram prensadas a $190^{\circ} \mathrm{C}$ para obtenção de filmes planos para a realização das análises de densidade, dureza Shore $D$, índice de fluidez (MFI), microscopia óptica (MO) e espectroscopia de infravermelho com transformada de Fourier (FTIR).

Tabela 1 - Materiais e proporções mássicas utilizadas na preparação dos compósitos.

\begin{tabular}{|c|c|}
\hline Materiais & Proporção mássica $(\mathrm{m} / \mathrm{m})$ \\
\hline MP & 100 \\
\hline MP/Mica 3\% & $97 / 3$ \\
\hline MP/Mica 10\% & $90 / 10$ \\
\hline $\mathrm{MP} / \mathrm{SiO}_{2} 3 \%$ & $97 / 3$ \\
\hline $\mathrm{MP} / \mathrm{SiO}_{2} 10 \%$ & $90 / 10$ \\
\hline
\end{tabular}

Fonte: As autoras.

\subsection{Análise de densidade}

Para os ensaios de densidade foi utilizada a Norma ASTM D792-2013. Utilizou-se o densímetro modelo DSL 910 (GEHAKA) e cinco corpos de prova de cada compósito preparado.

\subsection{Análise de dureza Shore D}

O ensaio de dureza Shore $D$ foi realizado nos materiais compósitos de acordo com a Norma ASTM D2240-2010, utilizando-se o durômetro Shore D (Type GS 702), em cinco pontos distintos de cada corpo de prova.

\subsection{Análise do Índice de Fluidez (MFI)}

Para a determinação do índice de fluidez (MFI) foi utilizado o equipamento medidor de índice de fluidez (CEAST- Quick Index) de acordo com a norma ASTM D1238-2013, a uma temperatura de $190^{\circ} \mathrm{C}$ e carga de 2,16 , tendo sido coletados e posteriormente pesados em balança analítica (Marte AY220) cinco espécimes de cada amostra.

\subsection{Microscopia óptica}

As amostras foram analisadas em microscópio óptico de alta capacidade da marca OLYMPUS, modelo BX51M, em aumentos variando de $50-200 \mathrm{x}$. 


\subsection{Espectroscopia de Infravermelho com Transformada de Fourier (FTIR)}

Para caracterizar a estrutura química das amostras, foram realizadas análises de FTIR (FTIR - modelo Nicolet 6700 da Thermo Scientific) no modo ATR, utilizando cristal de $\mathrm{ZnSe}$, na faixa de número de ondas de $600-4000 \mathrm{~cm}^{-1}$, com 32 varreduras por análise e resolução de $8 \mathrm{~cm}^{-1}$.

\section{RESULTADOS}

\subsection{Densidade, Dureza Shore D e MFI}

Os resultados dos ensaios de densidade, dureza e índice de fluidez estão apresentados na Tabela 2.

Tabela 2 - Resultados de Densidade, dureza e MFI dos materiais.

\begin{tabular}{|c|c|c|c|}
\hline Materiais & $\begin{array}{c}\text { Densidade } \\
\left(\mathrm{g} / \mathrm{cm}^{3}\right)\end{array}$ & $\begin{array}{c}\text { Dureza } \\
\text { (Shore D) }\end{array}$ & $\begin{array}{c}\text { MFI } \\
(g / 10 \mathrm{~min})\end{array}$ \\
\hline MP $100 \%$ & $0,841 \pm 0,116$ & $81,2 \pm 0,8$ & $0,26 \pm 0,07$ \\
\hline MP/Mica 3\% & $0,714 \pm 0,106$ & $80,2 \pm 2,2$ & $0,18 \pm 0,01$ \\
\hline MP/Mica 10\% & $0,744 \pm 0,164$ & $80,8 \pm 1,1$ & $0,22 \pm 0,02$ \\
\hline $\mathrm{MP} / \mathrm{SiO}_{2} 3 \%$ & $0,789 \pm 0,060$ & $80,0 \pm 0,1$ & $0,21 \pm 0,03$ \\
\hline $\mathrm{MP} / \mathrm{SiO}_{2} 10 \%$ & $0,472 \pm 0,018$ & $68,2 \pm 1,6$ & $0,04 \pm 0,01$ \\
\hline
\end{tabular}

Fonte: As autoras.

O valor de densidade encontrado para a madeira plástica está próximo aos valores encontrados na literatura (ALMEIDA, 2018), entre $0,700-0,800 \mathrm{~g} / \mathrm{cm}^{3}$. Considerando-se o desvio padrão, pode-se dizer que a adição de até 10 \% de mica não altera a densidade dos compósitos.

Em relação à sílica, o compósito $\mathrm{MP} / \mathrm{SiO}_{2} \quad 10 \%$ teve seu valor de densidade reduzido em mais de $50 \%$, sugerindo uma fraca interação entre a matriz e a $\mathrm{SiO}_{2} . \mathrm{A}$ fraca adesão faz com que haja um aumento dos espaços vazios na superfície fibra/matriz (BASTOS; LEÃO; PEREIRA, 2018), o que diminui o valor de densidade do compósito.

Os resultados de dureza mostram que a adição das cargas inorgânicas não foi suficiente para alterar a dureza dos compósitos, com exceção do compósito $\mathrm{MP} / \mathrm{SiO}_{2}$ $10 \%$ que teve seu valor de dureza reduzido, provavelmente devido a fraca interação, corroborando com o resultado de densidade.

A diminuição do índice de fluidez é associada usualmente a uma restrição na movimentação das cadeias devido ao aumento da carga, que torna o material mais viscoso, diminuindo assim o seu fluxo (MONSORES et al., 2017).

\subsection{Microscopia óptica}

A Figura 2 (a) - (d) exibe as imagens obtidas por microscopia óptica em aumento 
de 100 x. As partículas de $\mathrm{SiO}_{2}$ e mica podem ser visualizadas nas Figura 2 (a) e (b), respectivamente, onde é possível observar o formato lamelar de ambas e numa avaliação qualitativa permite verificar a faixa de tamanho das partículas, inferior a 100 $\mu \mathrm{m}$. As imagens da Figura 2 (c) e (d) mostram as partículas dispersas na matriz polimérica para os compósitos com $10 \%$ de cada carga.

Figura 2 - Imagens de microscopia óptica da sílica $\left(\mathrm{SiO}_{2}\right)(\mathrm{a})$, da mica (b) e dos compósitos $\mathrm{MP} / \mathrm{SiO}_{2} 10 \%$ (c) e MP/Mica10\% (d) em aumento de $100 \mathrm{x}$.
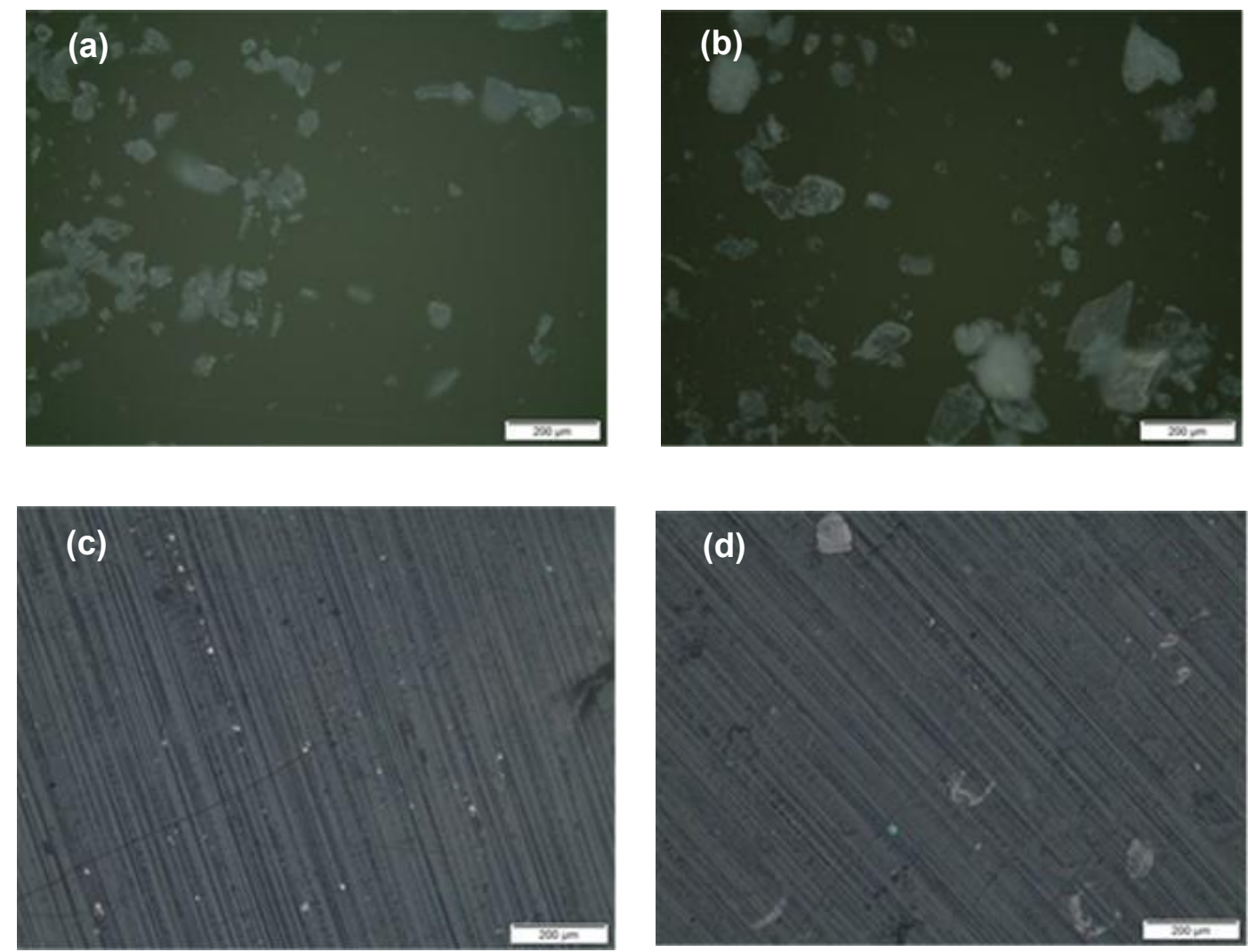

Fonte: As autoras.

\subsection{FTIR}

A madeira plástica apresenta picos típicos do PEAD. O PEAD é formado pela repetição do grupo $-\left(\mathrm{CH}_{2}\right)-$. O espectro da MP mostra as três bandas de absorção associadas aos três modos de vibração da ligação $\mathrm{C}-\mathrm{H}$, cujos valores dos números de onda se situam nas seguintes regiões espectrais: valência ou "stretching" entre 2950 e $2850 \mathrm{~cm}^{-1}$; pêndulo ou "bending" entre 1350 e $1450 \mathrm{~cm}^{-1}$; torção ou "rocking" aproximadamente em $700 \mathrm{~cm}^{-1}$ (TORRES, 2007; ALMEIDA, 2018).

Cada banda do PEAD é formada por dois picos, devido ao fato de que a frequência de vibração de uma ligação depende do meio que a envolve. Assim, para o polietileno, cada pico corresponde a uma configuração C-H particular. Uma banda é típica das ligações dentro da cadeia polimérica, enquanto a outra corresponde à ligação $\mathrm{C}-\mathrm{H}$ nas extremidades da cadeia polimérica. A amplitude relativa de um pico sobre o outro 
depende da importância relativa de cada tipo de ligação e de seu grau de vibração. $O$ PEAD também apresenta as ligações $C-C$ que são normalmente observadas com pico(s) de absorbância em um número de onda na região entre $1000-1250 \mathrm{~cm}^{-1}$ (TORRES, 2007; ALMEIDA, 2018). A figura 3 mostra os espectros de FTIR-ATR dos materiais obtidos.

Figura 3 - Espectros de FTIR-ATR dos materiais obtidos.

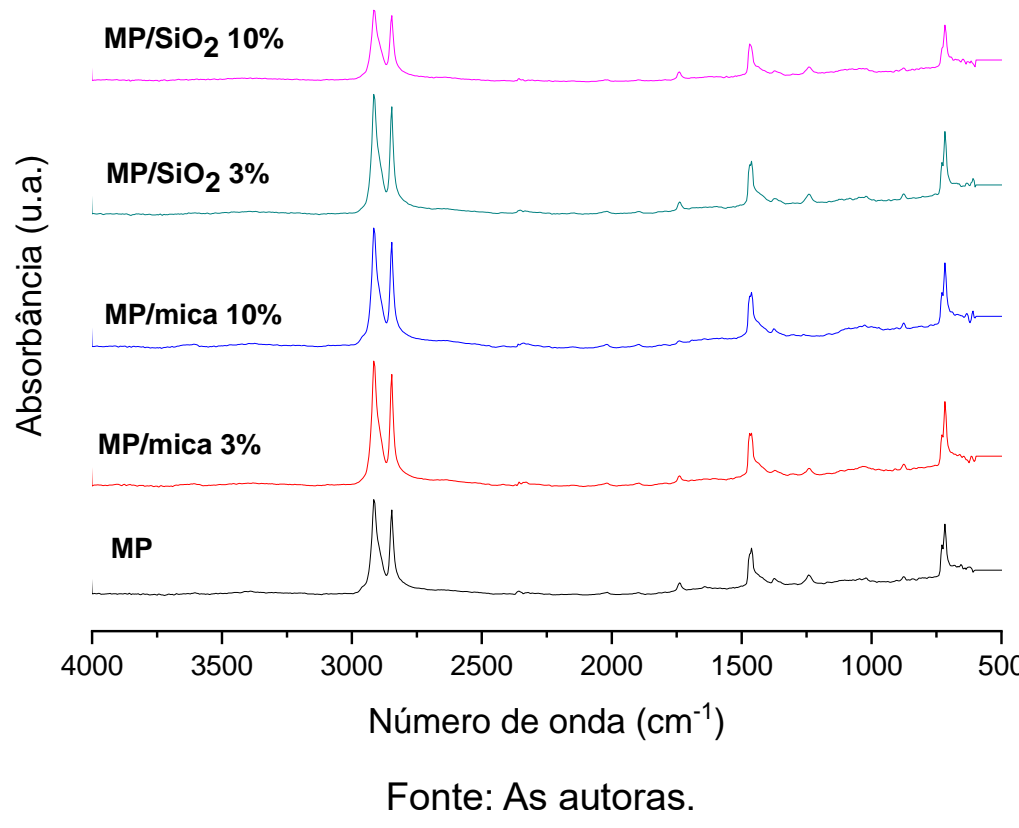

De acordo com a literatura, as vibrações de minerais do grupo das mica podem ser separadas em uma região vibracional de grupos hidroxila e em uma região vibracional compreendendo ligações dos tetraedros $\mathrm{Si}(\mathrm{Al}) \mathrm{O}_{4}$, dos cátions coordenados octraedricamente, e dos cátions intercamadas altamente coordenados. Esta separação é completa na região entre $3700-3550 \mathrm{~cm}^{-1}$ ( $\mathrm{OH}$ "stretching") de alta energia. As bandas de absorção na região entre $3700-3550 \mathrm{~cm}^{-1}$ podem ser atribuídas à valência ou "stretching" da ligação Si-O presente na estrutura cristalina da mica.

A vibração da ligação Si-O na região entre $600-300 \mathrm{~cm}^{-1}$, normalmente, apresenta um acoplamento entre "stretching" e "bending" dos octraedros cátion-oxigênio situados em uma região espectral similar. As vibrações dos cátions intercamadas estão localizadas na faixa entre $400-50 \mathrm{~cm}^{-1}$ que não estão presentes neste espectro (BERAN, 2002).

Outros picos presentes no espectro podem ser atribuídos a aditivos utilizados para melhorar o fluxo do polímero pós-consumo durante o reprocessamento do material (ALMEIDA, 2018). 


\section{CONCLUSÂO}

A mica não causou grandes alterações na estrutura do polímero. Porém, na adição de $10 \%$ de $\mathrm{SiO}_{2}$ notou-se a redução das propriedades físicas, o que pode ser atribuído a uma falta de interação para esse sistema, que pode ter causado uma desorganização da estrutura molecular. A processabilidade das amostras diminui com a inserção das cargas, como mostram os resultados de MFI, pois a carga inorgânica pode causar obstruções mecânicas que dificultam o processamento. Os espectros de FTIR mostram bandas de absorção características do PEAD e de ligações Si-O presentes tanto na mica quanto na sílica. Pode-se inferir que não há interação química, apenas ligação de van der Walls entre os materiais após o processamento. O melhor resultado do presente estudo pode ser atribuído ao compósito $\mathrm{MP} /$ mica $10 \%$, que mostrou melhores propriedades, possivelmente pela melhor interação da MP com a mica.

\section{AGRADECIMENTOS}

FAPERJ, CETEM, COMLURB.

\section{REFERÊNCIAS}

ALMEIDA, P. O. Compósitos de madeira plástica reforçada com mica muscovita. 2018. 77f. Dissertação (Mestrado Profissional em Ciência e Tecnologia dos Materiais) Centro Universitário Estadual da Zona Oeste - UEZO, Rio de Janeiro, 2018.

BASTOS, D.C.; LEÃO, A.G.; PEREIRA, P.S.C. Characterization of PP postconsume/banana fiber composites. Acta Scientiae \& Technicae, v. 6, n. 1, p. 19-22, 2018.

BERAN, A. Infrared Spectroscopy of Micas. Reviews in Mineralogy and Geochemistry, v. 46, n. 1, p. 351-369, 2002.

CHENG, W. et al. Preparation of silica/polyacrylamide/polyethylene nanocomposite via in situ polymerization. Materials Letters, v. 61, n. 14-15, p. 3193-3196, Junho. 2007.

CRUZ, S. A. et al. "Polímeros reciclados para contato com alimentos". Polímeros, v. 21, n. 4, p. 340-345, 2011.

DIAS, B. Z.; ALVAREZ, C. E. de. Mechanical properties: wood lumber versus plastic lumber and thermoplastic composites. Ambiente Construído, v. 17, n. 2, p. 201-219, 2017.

ESCÓCIO, V. A. et al. Efeito do Envelhecimento nas Propriedades Mecânicas e Dinâmico-Mecânicas de Composições de Borracha Natural com Mica, Polímeros: Ciência e Tecnologia, v. 14, n. 1, p.13-16. 2004.

FELIX, A. H. O. Compósitos de polipropileno com fibra de vidro utilizando viniltrietoxisilano como agente de adesão. 2002. 129f. Dissertação (Mestrado em Engenharia Química) - Universidade Federal do Rio Grande do Sul, Porto Alegre, 2002. 
FONSECA; L. T. R., Estudo do desenvolvimento de compósitos de PEAD com $\mathrm{SiO}_{2}$. 2015. 44f. Trabalho de Conclusão de Curso (Tecnologia em Polímeros) - Centro Universitário Estadual da Zona Oeste (UEZO), Rio de Janeiro, 2015.

LI-YU, L.; DAE-EUN, K. Tribological properties of polymer/silica composite coatings for microsystems applications. Tribology International, v. 44, n. 12, p. 1926-1931, Novembro. 2011.

MARINELLI, A. L.; MONTEIRO, M. R.; AMBROSIO, J. D.; et al, Desenvolvimento de Compósitos Poliméricos com Fibras Vegetais Naturais da Biodiversidade: Uma Contribuição para a Sustentabilidade Amazônica, Polímeros: Ciência e Tecnologia, v. 18, p.92-99, 2008.

MARTINS, S. Resíduo da fabricação de catalisadores de FCC utilizado como retardante de chamas em PEAD reciclado. 2017. 123f. Dissertação (Mestrado Profissional em Ciência e Tecnologia dos Materiais) - Centro Universitário Estadual da Zona Oeste - UEZO, Rio de Janeiro, 2017.

MATOS, M. C. C. Revisão sobre o Poli(tereftalato de etileno), com ênfase na aplicação em garrafas para bebidas. 2015. 63f. Dissertação (Mestrado Profissional em Ciência e Tecnologia dos Materiais) -Centro Universitário Estadual da Zona Oeste UEZO, Rio de Janeiro, 2015.

MONSORES, K. G. C. et al. Materiais Compósitos a base de PP e muscovita. Revista Iberoamericana de Polímeros, v. 18, n. 6, p. 301-309, 2017.

NICOLAU, G. da S. Compósito de mica muscovita com polipropileno: Estudo da influência do uso de compatibilizante. 2017. 49f. Trabalho de Conclusão de Curso (Tecnologia em Polímeros) - Centro Universitário Estadual da Zona Oeste (UEZO), Rio de Janeiro, 2017.

OLIVEIRA, E. M. R. de; COSTA, R. A. Dossiê Técnico - Madeira Plástica. 29 p. Instituto Euvaldo Lodi - IEL/BA. SBRT - Serviço Brasileiro de Respostas Técnicas. 2013.

OMAR, M. F.; AKIL, H. M.; AHMAD, Z. A. Static and dynamic compressive properties of mica/polypropylene composites, Materials Science and Engineering A, v. 528, 15671576, Janeiro. 2011.

PALZA, H.; VERGARA, R.; ZAPATA, P. Composites of polypropylene melt blended with synthesized silica nanoparticles. Composites Science and Technology, v. 71, n. 4, p.535-540, February, 2011.

PASSOS, A. A. et al. Obtenção de nanocompósito de EVA/SíLICA e caracterização por ressonância magnética nuclear no estado sólido. Polímeros, v. 21, n. 2, p. 98-102, Maio, 2011.

RAMOS, S. M. L. S. et al. Efeitos da estabilização do Polipropileno nas propriedades térmicas, mecânicas e termo-mecânicas de compósitos de Polipropileno/Atapulgita, Polímeros: Ciência e Tecnologia, v.3, n. 4, p. 26-31, 1993.

RAMOS FILHO, F. G. et al. Thermal stability of nanocomposites based on polypropylene and bentonite, Polymer Degradation and Stability, v. 89, n 3, p. 383392, 2005. 
RASHID, E. S. A. et al. Effect of ion exchange treatment on the properties of muscovite filled epoxy composite, Applied Clay Science, v. 52, n. 3, p. 295-300, 2011.

REZENDE, W. C. de. Avaliação da logística reversa e da reciclagem da madeira plástica utilizada pela COMLURB. 2015. 100f. Dissertação (Mestrado Profissional em Ciência e Tecnologia dos Materiais) - Centro Universitário Estadual da Zona Oeste - UEZO, Rio de Janeiro, 2015.

ROMÃO, W.; FRANCO, M. F.; BUENO, M. I. M. S.; Distinguishing between virgin and post-consumption bottle-grade poly (ethylene terephthalate) using thermal properties, Polymer Testing, v. 29, p. 879-885, 2010.

SANTOS, S. F.; ANDRADE, M. C.; SAMPAIO, J. A.; et al; Thermal Study of $\mathrm{TiO}_{2}-\mathrm{CeO}_{2}$ yellow ceramic pigment obtained by the Pechini method, Journal of Thermal Analysis and Calorimetry, v. 87, n. 3, p. 743-746, 2007.

SANTOS, S. F.; FRANÇA, S. C. A.; OGASAWARA, T. Method for grinding and delaminating muscovite, Mining Science and Technology, v. 21, n. 1, p.7-10, 2011.

TORRES, A. A. T. Envelhecimento físico-químico de tubulações de polietileno de alta densidade empregadas em redes de distribuição de derivados de petróleo. 2007. 180f. Dissertação (Mestrado em Ciência dos Materiais e Metalurgia) - Pontifícia Universidade Católica, Rio de janeiro, 2007.

UNO, H. et al. Preparation and mechanical properties of exfoliated mica-polyamide 6 nanocomposites using sericite mica, Applied Clay Science, v. 46, p. 81-87, 2009.

VERBEEK, C. J. R., Highly filled polyethylene phlogopite composites, Materials Letters, v. 52, n. 6, p.453-457, 2002. 